Methodology article

\title{
Inhibition of cell growth and invasion by epidermal growth factor-targeted phagemid particles carrying siRNA against focal adhesion kinase in the presence of hydroxycamptothecin Xiu-Mei Cai ${ }^{1}$, Hai-Long Xie ${ }^{2}$, Ming-Zhu Liu ${ }^{1}$ and Xi-Liang Zha*1
}

\author{
Address: ${ }^{1}$ Department of Biochemistry and Molecular Biology, Shanghai Medical College, Fudan University, Shanghai, PR China and ${ }^{2}$ Institute of \\ Cancer Research, South China University, Hengyang, PR China \\ Email: Xiu-Mei Cai - caixm06@gmail.com; Hai-Long Xie -xhl0078@sina.com; Ming-Zhu Liu - fdu_liumingzhu@hotmail.com; Xi- \\ Liang Zha* - caixioum@163.com \\ * Corresponding author
}

Published: 18 September 2008

BMC Biotechnology 2008, 8:74 doi:10.1 186/1472-6750-8-74
Received: 2 April 2008

Accepted: 18 September 2008

This article is available from: http://www.biomedcentral.com/1472-6750/8/74

(C) 2008 Cai et al; licensee BioMed Central Ltd.

This is an Open Access article distributed under the terms of the Creative Commons Attribution License (http://creativecommons.org/licenses/by/2.0), which permits unrestricted use, distribution, and reproduction in any medium, provided the original work is properly cited.

\begin{abstract}
Background: Previous studies demonstrated the EGF-targeted phagemid particles carrying siRNA against Akt could be expressed efficiently in the presence of hydroxycamptothecin (HCPT). However, no significant cell growth inhibition was obtained. This study was to further investigate whether the EGF-targeted phagemid particles carrying siRNA would be a promising tool for anticancer siRNA delivery.
\end{abstract}

Results: We found that pSi4.I-siFAK phagemid particles could significantly inhibit the expression of focal adhesion kinase in the HCPT-treated cells. Moreover, we also observed that the particles could potently suppress cell growth and cell invasion.

Conclusion: These results indicated that EGF-targeted phagemid particles might be a promising tool for anti-cancer siRNA delivery in the presence of HCPT.

\section{Background}

Small interfering RNA (siRNA) molecules are capable of interrupting the translation of a specific protein by inducing post-transcriptional gene silencing. It is a promising method for silencing therapeutic target genes. A variety of delivery systems are proposed for the delivery of siRNA into cells in vitro and in vivo. Since phage-based vectors do not exhibit natural tropism towards mammalian cells and can be genetically modified for specific applications, modified phage-based vectors are an attractive alternative strategy for gene delivery. They have been successfully modified to deliver genes to target cells by the effective use of targeting ligands such as growth factors, antibodies, and viral capsid proteins [1-7]. To increase the density of ligand display on the phages, an epidermal growth factor (EGF)-modified helper phage genome M13EGFKO7CT was established, which could produce EGF-targeted phagemid particles [8]. The phagemid particles could deliver reporter genes into target cells; however, the efficiency of delivery was limited [8]. A topoisomerase I inhibitor such as camptothecin or hydroxycamptothecin (HCPT) could substantially enhance the transduction of the phagemid gene delivery particles $[1,9]$. The recent studies showed that the cell-targeted phagemid particles could efficiently deliver siRNA against Akt into cell in the presence of HCPT [10]. But, no significant growth inhibi- 
tion was observed. Thus, to be an effective anti-cancer siRNA delivery vector, more studies should be performed, such as carrying siRNA against other oncogenes.

Focal adhesion kinase (FAK), a non-receptor tyrosine kinase, has been implicated in several cellular processes such as proliferation, apoptosis, motility, and invasion. Increased expression of FAK has been found in various malignant tumors, including tumors derived from the lungs, breasts, head and neck, and ovaries [11-14]. Therefore, FAK is recognized as an important therapeutic target in the treatment of cancer. Delivery of siFAK by lipofectamine could significantly block the expression of FAK and trigger cell death and block cell migration [15]. But, the siFAK could not be delivered to target cells. To further investigate whether the EGF-targeted phagemid particles in combination with RNA interference (RNAi) would represent an effective therapeutic approach, we used phagemid particles carrying siRNA against FAK to infect H1299 cells and examined the therapeutic potential of this approach.

\section{Results and Discussion}

Previous studies showed that the cell-targeted phagemid particles were efficient siRNA delivery vectors in the pres- ence of HCPT and they could efficiently deliver siRNA against Akt into targeted cells in the presence of HCPT [10]. But, no significant growth inhibition was observed. Thus, to be an effective anti-cancer siRNA delivery vector, more studies should be performed, such as carrying siRNA against other oncogenes. In this study, we made phagemid particles carrying siRNA against FAK to infect H1299 cells and examined the therapeutic potential of this approach. First, the short hairpin RNA (shRNA) against FAK was subcloned into pSi4.1CMV-f1, thus forming pSilencer4.1-siFAK (pSi4.1-siFAK) (Fig. 1A). Then, we purified ssDNA from phagemid particles to analyze the ratio of phagemids to helper phage genomes packaged in the phagemid particles. The results indicated that almost all the DNA packaged comprised phagemids (Fig. 1B). Previously, the modified helper phage genome (plasmid) M13EGFKO7CT was created to produce EGF-targeted phagemid particles $[8,10]$. The M13EGFKO7CT plasmid was used to package pSi4.1-siFAK phagemid particles, following which the phagemid particles displayed the EGF ligand. In the immunocytochemical assay, we found that H1299 cells showed a strong positive EGFR immunoreactivity, while very light immunostaining was observed in the U87 cells that were used as negative controls (Fig. 2A). Therefore, we infected H1299 cells with pSi4.1-siFAK
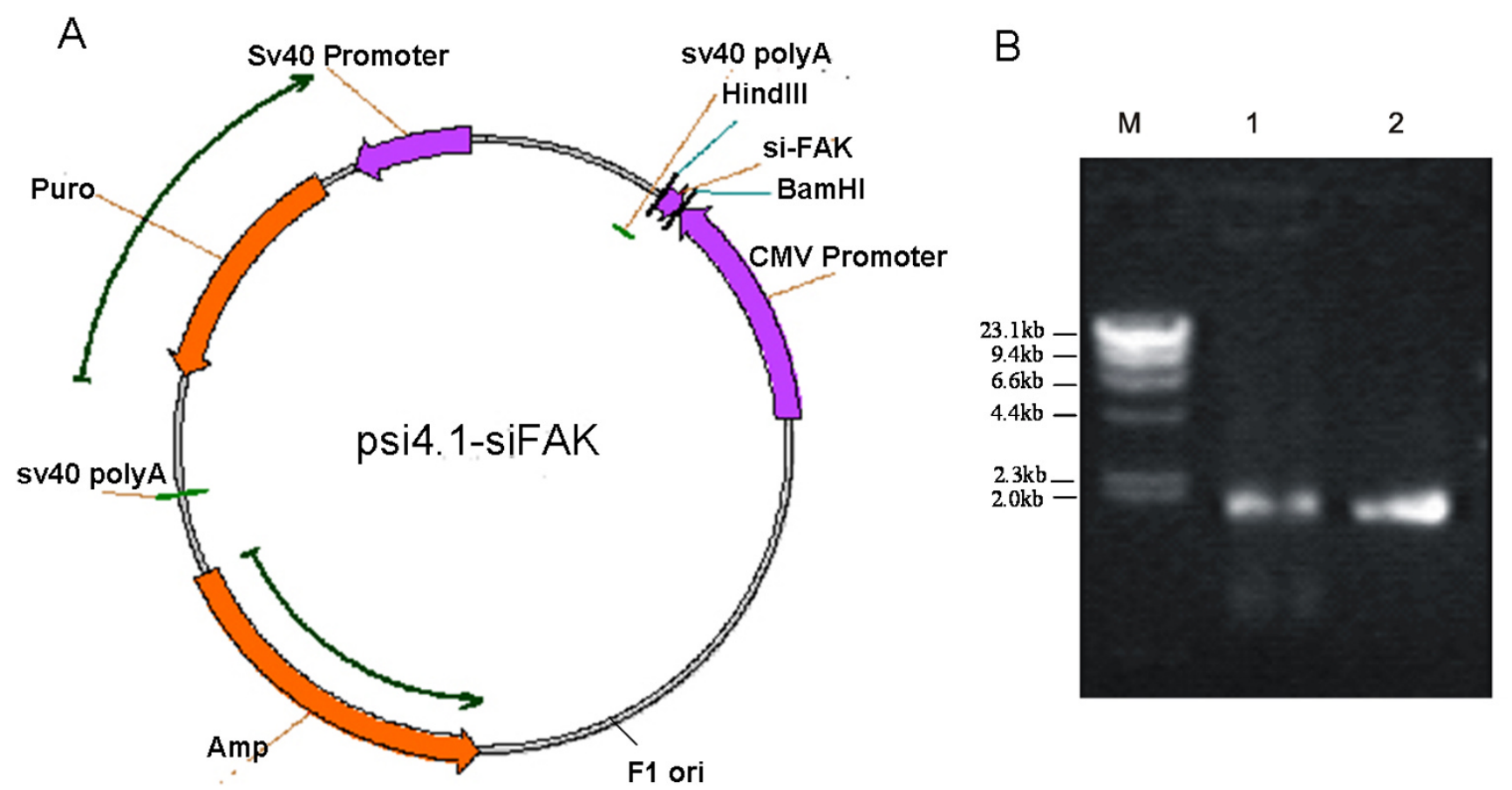

\section{Figure I}

The map of pSi4.I-siFAK phagemid particle and analysis of the ssDNA released from phagemid particles. A: PSi4. I-siFAK phagemid particle was constructed as follows: the shRNA against FAK was inserted into the modified pSilencer4.I vector digested with BamHI and HindIII. B: ssDNA was purified from phagemid particles to analyze the ratio of phagemids to helper phage genomes packaged in the phagemid particles. The results indicated that almost all the DNA packaged comprised phagemids. Lane M: $\lambda$ DNA/HindIII; Lane I: pSi4.I-siFAK phagemid particles; Lane 2: pSi4.I-simock phagemid particles. 
phagemid particles. Western blotting assay showed that the pSi4.1-siFAK plasmid transfected by lipofectamine could significantly block the expression of FAK. This was not observed in cells transduced with pSi4.1-siFAK phagemid particles without HCPT treatment. Surprisingly, in HCPT treated-cells, the pSi4.1-siFAK phagemid particles could inhibit FAK expression to a great extent. Inhibition of FAK expression was not found in the cells infected with mock phagemid particles (Fig. 2B). Taken together, the vectors could deliver siRNA to human carcinoma cells efficiently in the presence of HCPT. HCPT had been shown to increase the efficiency of transduction of the phagemid vectors $[1,9,16]$. However, the mechanism by which HCPT increased transgene expression was not fully understood [1,9]. It was thought to involve the activation of the host cell repair machinery in response to DNA damage $[1,16,17]$; however, further studies are required to confirm this.
A series of experiments were performed to determine whether the pSi4.1-siFAK phagemid particles could arrest H1299 cell growth. Cell growth was monitored by the MTT assay, and it was observed that all the cells grew at a similar rate at 0 and $24 \mathrm{~h}$. However, a great difference began to appear at 48 and $72 \mathrm{~h}$. In the presence of HCPT, the inhibitory rates were approximately $12-19 \%$ in parent and mock cells, compared with parent cells without HCPT. Amazingly, the inhibitory rates reached approximately $52-61 \%$ in HCPT-treated cells infected with pSi4.1-siFAK phagemid particles, compared with the control cells. No significant growth inhibition was found in the cells infected with the mock vector or pSi4.1-siFAK phagemid particles in the absence of HCPT (Fig. 3A). In addition, we examined the growth arrest of the pSi4.1siFAK phagemid particles at the 3-dimensional level. In the colony-forming ability assay, the number of colonies of pSi4.1-siFAK phagemid particles decreased by almost

\section{A}
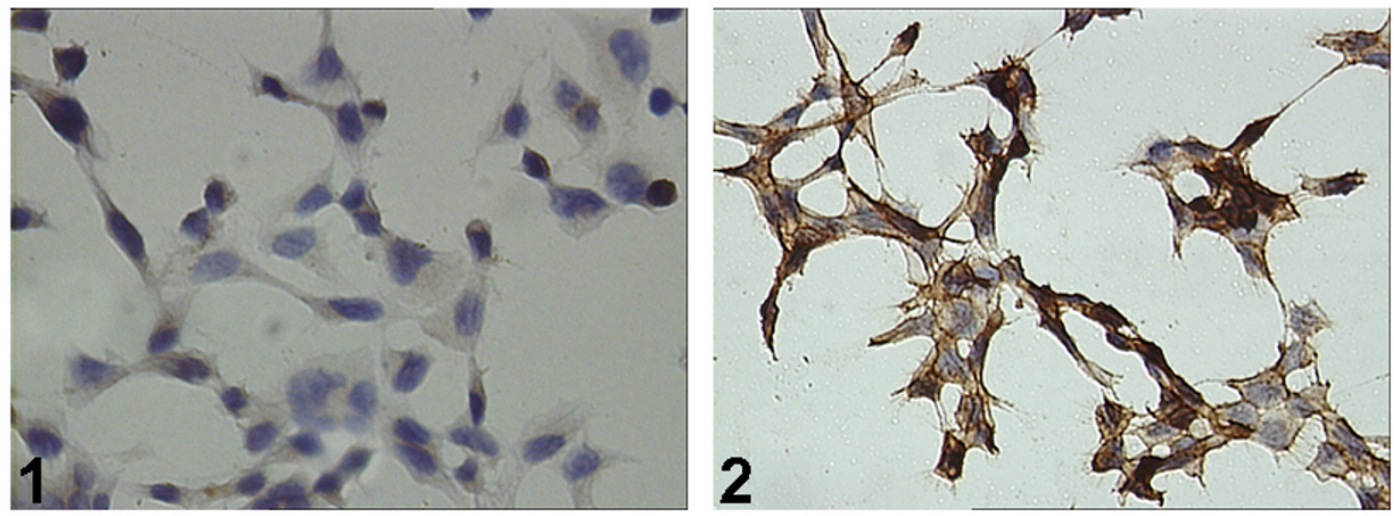

B
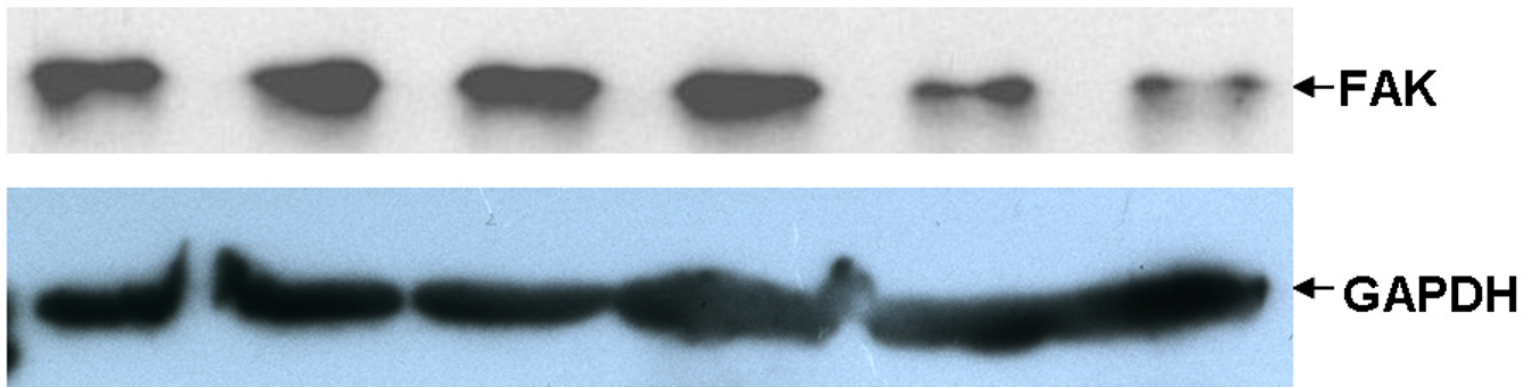

Figure 2

Immunocytochemistry assay of EGFR expression and Western-blot analysis of the specific FAK gene silencing by EGF-targeted phagemid particles mediated RNA interference. A: In the immunocytochemical assay, HI299 cells showed a strong positive EGFR immunoreactivity, while very light immunostaining was observed in the U87 cells that were used as negative controls. I, HI299; 2, U87. B: HI299 cells were infected with PSi4.I-siFAK phagemid particles. In HCPT treated-cells, the pSi4. I-siFAK phagemid particles could inhibit FAK expression to a great extent. I, HI299; 2, HI299 infected with pSi4. I-simock phagemid particles; 3, HI299 infected with pSi4.I-simock phagemid particles in the presence of $2.5 \mu \mathrm{M}$ HCPT; 4, HI 299 infected with pSi4. I-siFAK phagemid particles; 5, HI 299 infected with pSi4.I-siFAK phagemid particles in the presence of $2.5 \mu \mathrm{M}$ HCPT; 6, HI299 transfected with pSi4. I-siFAK using Lipofectamine 2000. 
$54 \%$ in the presence of HCPT, compared with the control cells. In contrast, cells in other groups exhibited little change in the number of colonies (Fig. 3B). These data suggested that the treatment of pSi4.1-siFAK phagemid particles could dramatically reduce cell viability in the presence of HCPT.

Furthermore, we quantified the effect of pSi4.1-siFAK phagemid particles on cell invasion. In the transwell invasion assay, the number of pSi4.1-siFAK phagemid particles transduced-cells (HCPT treatment) invading through the membrane coated with ECM gel was less than that of the control cells (Fig. 4A). The cell invasion was markedly reduced by approximately $50 \%$ in pSi4.1-siFAK phagemid particles transduced-cells (HCPT treatment), compared with the control cells; the other groups however showed no obvious change (Fig. 4B). Thus, the above data indicated that the transfection of H1299 cells with pSi4.1siFAK phagemid particles and the HCPT treatment could dramatically inhibit cell invasion.

RNAi has revolutionized the biological sciences because it can selectively silence messenger RNA (mRNA) expres-

A
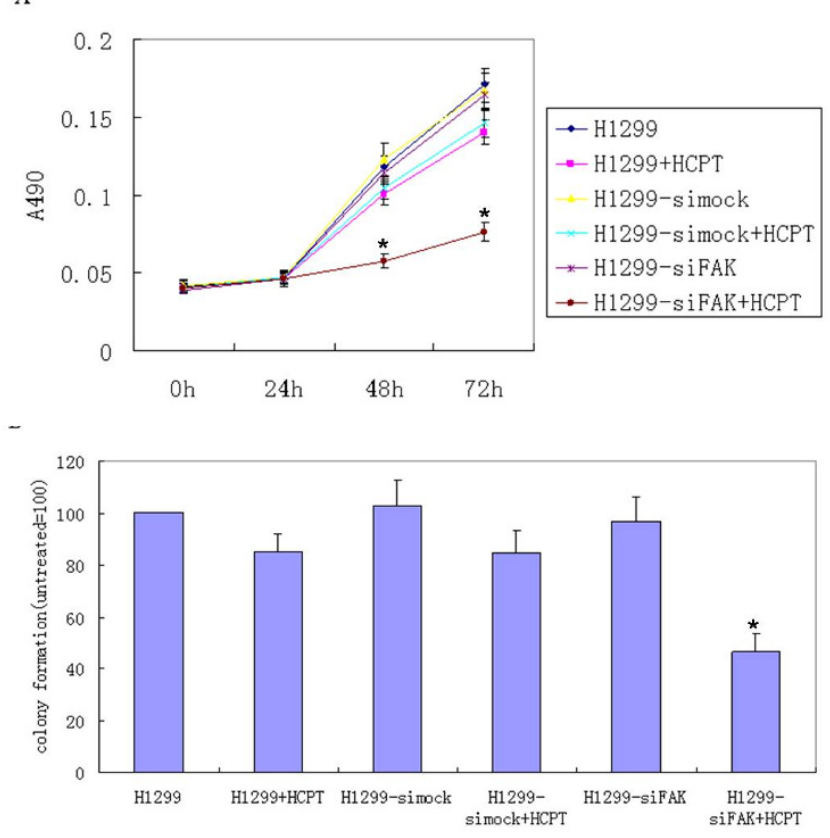

Figure 3

Phagemid particles of pSi4.I-siFAK could inhibit

HI 299 cell growth. A: MTT assay. B: Colony-Forming Ability Assay. The results shown were representative of at least three independent experiments. The HCPT-treated cells that were infected with pSi4.I-siFAK phagemid particles exhibited a significant inhibition of proliferation $(*, p<0.0$ I) compared with $\mathrm{HI} 299$ cells. sion. However, the delivery of this RNA into target cells represents the main barrier for using siRNA as a novel drug against tumor targets. Since filamentous phages only showed tropism for cells that expressed the appropriate receptors, this tropism could be conferred to phage particles by the expression of a targeting ligand on the phage coat $[3,4,6]$. Therefore, phages would be an attractive alternative strategy for siRNA delivery. This study demonstrated that EGF-targeted phagemid particles in combination with RNAi and the HCPT treatment represent a new therapeutic approach for silencing oncogenes. However, in order to act as a cancer gene-delivery vector, phage vectors should display other targeting ligands to increase their specificity for different types of tumors.

\section{Conclusion}

Therefore, phages would be an attractive alternative strategy for siRNA delivery. This study demonstrated that EGFtargeted phagemid particles in combination with RNAi and the HCPT treatment represent a new therapeutic approach for silencing oncogenes. However, in order to act as a cancer gene-delivery vector, phage vectors should display other targeting ligands to increase their specificity for different types of tumors.

\section{Methods \\ Reagents}

Dulbecco's Modified Eagle's Medium (DMEM), Dulbecco's phosphate-buffered saline and fetal bovine serum (FBS) were obtained from Invitrogen (Grand Island, USA). Restriction endonucleases were obtained from TaKaRa Biotechnology (Dalian, China).

\section{Cell culture}

H1299 (human lung carcinomas) cells and U87 (human glioblastoma) cells were cultured at $37^{\circ} \mathrm{C}$ in DMEM containing $10 \%$ FBS in a humidified atmosphere containing $5 \% \mathrm{CO} 2$.

\section{Plasmid construction}

The modified pSilencer4.1 plasmid was obtained from Dr. Z. Li (Shanghai Jiao Tong University, Shanghai, China). FAK siRNA (target sequence, 5'-GAACCTCGCAGTCATTTAT-3') has been proven to be effective for inhibiting FAK [18]. A 59-nt oligo-DNA duplex (5'AGCTTGAACCTCGCAGTCATTTATTTCAAGA-

GAATAAATGACTGCGAG GTTCTTTTTTG-3'/5'GATCCAAAAAAGAACCTCGCAGTCATTTATTCTCTTG AAATAAATGACTGCGAGGTTCA-3') was inserted into the pSilencer4. 1 vector digested with BamHI and HindIII. The mock siRNA sequence is 5'-GTCTCCGAACGTGTCACGT3' [18]. Another 59-nt oligo-DNA duplex (5'AGCTTGTCTCCGAACGTGTCACGTTTCAAGAGAACGTGACACGTTCGGAGACTTTTTTG-3'/5'GATCCAAAAAAGTCTCCGAACGTGTCACGTTCTCTT- 
A
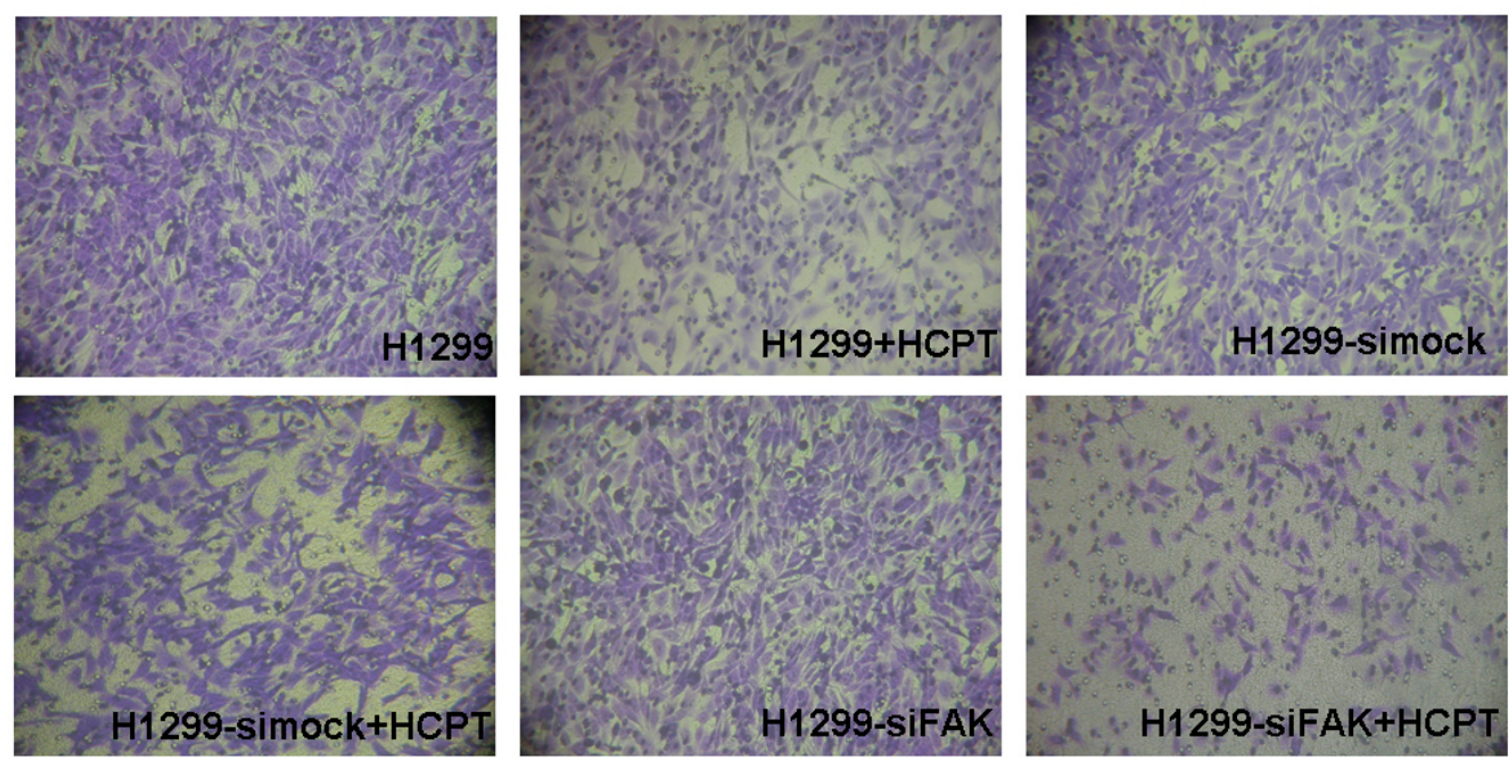

B

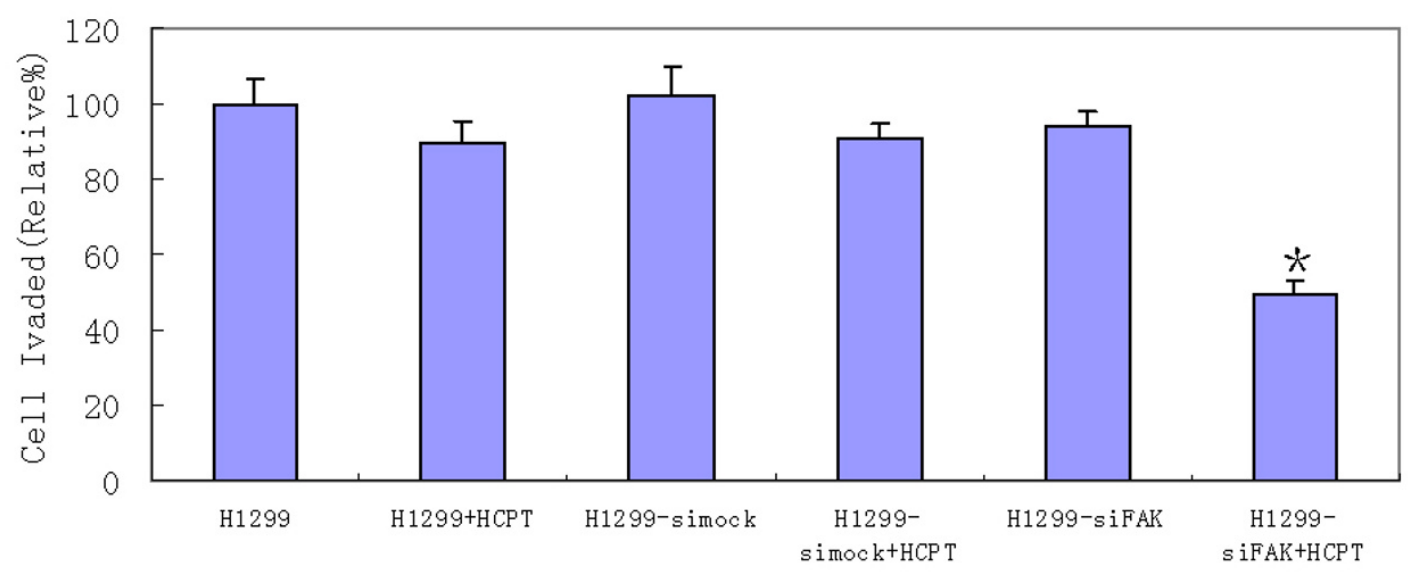

Figure 4

Phagemid particles of pSi4. I-siFAK could inhibit HI 299 cell invasion. A: Cells that invaded after 36 hr through ECMGel-coated transwell inserts were stained with crystal violet stain. B: Cell invasion assay. The HCPT-treated cells that were infected with pSi4. I-siFAK phagemid particles exhibited a significant inhibition of invasion (*, p < 0.0I) compared with HI299 cells.

GAAACGTGACACGTTCGGAGACA-3') was inserted into the modified pSilencer4.1 vector digested with BamHI and HindIII.

\section{Preparation of phagemid particles}

Briefly, M13KO7EGFCT was transformed into Escherichia coli to create LMP cells [8]. The phagemid carrying siFAK was transformed into the LMP cells. The cells were then plated on Luria-Bertani (LB) agar containing $70 \mu \mathrm{g} / \mathrm{mL}$ kanamycin and $50 \mu \mathrm{g} / \mathrm{mL}$ ampicillin and incubated at $37^{\circ} \mathrm{C}$ overnight. A cell clone was picked up and transferred into $1 \mathrm{~L}$ of LB solution containing $70 \mu \mathrm{g} / \mathrm{mL}$ kan- amycin and $50 \mu \mathrm{g} / \mathrm{mL}$ ampicillin. After shaking at $37^{\circ} \mathrm{C}$ for $15 \mathrm{~h}$, the supernatant of the culture was collected and the phagemid particles were purified with polyethylene glycol (PEG)/ $\mathrm{NaCl}$ precipitation. Then, they were quantified by ELISA as previously described [19].

\section{Purification of single-stranded DNA from phagemid particles}

Single-stranded DNA (ssDNA) was extracted from the phagemid particles by using the Ph.D.-12 phage display peptide library kit (New England Biolabs, USA) according to the manufacturer's protocol. Briefly, the phagemid par- 
ticles were precipitated by PEG/NaCl. The pellet was then suspended in iodide buffer. Ethanol $(250 \mathrm{ml}$ in total $)$ was added to the buffer, and the pellet was incubated in it for $10 \mathrm{~min}$. The pellet was collected after centrifugation at 12 $000 \times g$ for $10 \mathrm{~min}$. It was finally dissolved in $30 \mu \mathrm{L}$ TrisEDTA (TE) buffer (10 mM Tris-HCl [pH 8.0] and $1 \mathrm{mM}$ EDTA) and analyzed by agarose gel electrophoresis.

\section{Immunocytochemistry}

Monolayer cells were grown on glass cover slips and fixed with $4 \%$ paraformaldehyde. Endogenous peroxidase activity was quenched with $2 \%$ hydrogen peroxide in methanol for $45 \mathrm{~min}$. After the cells were blocked with 5\% normal serum for $30 \mathrm{~min}$, they were incubated with primary antibodies to the epidermal growth factor receptor (EGFR) (Cell Signaling Technology, MA) diluted to 1:500 in phosphate-buffered saline (PBS) for $1 \mathrm{~h}$ at room temperature. Then, the cells were rinsed and incubated with horseradish peroxidase (HRP)-conjugated secondary antibodies (Watson Biotech, China) diluted to 1:500 in PBS for $1.5 \mathrm{~h}$ at room temperature. The reaction was developed with diaminobenzidine (Dako, Japan), and then the slides were counterstained with Mayer's hematoxylin. After a final wash, the slides were mounted and the cells were examined using an Olympus photomicroscope (400× magnification).

\section{In vitro phagemid particle transfection}

The cells were plated onto 24 -well plates at a density of 10 000 cells per well $24 \mathrm{~h}$ prior to the addition of phage particles. The phages were added at $10^{11} \mathrm{pfu} / \mathrm{mL}$ and incubated with the cells for $48 \mathrm{~h}$ at $37^{\circ} \mathrm{C}$ in complete media. Then, the medium was removed and the cells were incubated in fresh medium containing $2.5 \mu \mathrm{M}$ HCPT for $6 \mathrm{~h}$ at $37^{\circ} \mathrm{C}$. Following this, the medium was replaced with fresh medium, and the cells were incubated for $18 \mathrm{~h}$ at $37^{\circ} \mathrm{C}$. All the transfections were performed in triplicate at least 2 times.

\section{Western blot analysis}

In total, $10^{6} \mathrm{H} 1299$ cells transfected with a variety of phagemid particles were lysed after the HCPT treatment. The cells were washed with PBS and lysed in a buffer containing $50 \mathrm{mM}$ Tris (pH 7.5), $5 \mathrm{mM}$ EDTA, $300 \mathrm{mM} \mathrm{NaCl}$, $0.1 \%$ Igepal, $0.5 \mathrm{mM} \mathrm{NaF}, 0.5 \mathrm{mM} \mathrm{Na}_{3} \mathrm{VO}_{4}, 0.5 \mathrm{mM}$ phenylmethylsulfonyl fluoride, and an antiprotease mixture. Equal amounts of protein were loaded on a SDS-PAGE and transferred onto a nitrocellulose membrane. They were incubated with specific primary antibodies and then with HRP-conjugated secondary antibodies. Proteins were visualized by fluorography using an enhanced chemiluminescence system (Pierce Biotechnologies, USA). The anti-FAK antibody (Santa Cruz, CA) was used in 1:1000 dilutions. The monoclonal antibody to GAPDH was purchased from Kang-Chen Biotech (Shanghai, China). The secondary antibody conjugated with HRP was purchased from Watson Biotech (Shanghai, China).

\section{MTT assay}

After the HCPT treatment, H1299 cells transfected with a variety of phagemid particles were seeded onto a 96-well plate overnight in DMEM containing 10\% FBS, and then grown for $0,24,48$, and $72 \mathrm{~h}$. Methyl thiazolyl tetrazolium (MTT) $(20 \mu \mathrm{L})$ solution $(5 \mathrm{mg} / \mathrm{mL}$ in PBS) was added to each well and incubated for $5 \mathrm{~h}$ at $37^{\circ} \mathrm{C}$. The solution was removed and $200 \mu \mathrm{L}$ of dimethylsulfoxide (DMSO) was added to each well. These plates were vibrated gently for $10 \mathrm{~min}$; they then underwent detection in the universal microplate reader at $490 \mathrm{~nm}$.

\section{Colony-forming ability assay}

The efficiency of colony formation was assayed in 35- $\mathrm{mm}$ dishes prepared with a lower layer of $0.8 \%$ agar (GIBCO/ BRL) overlaid with $0.3 \%$ agar containing $2 \times 10^{4}$ suspended cells. After 5 days, growth was estimated under a Nikon inverted phase-contrast microscope, and individual colonies of more than 50 cells were counted [20].

\section{Transwell invasion assay}

Polymerized gels were prepared by neutralization of extracellular matrix (ECM) gel (Sigma, USA) with cold DMEM. Cells in DMEM with 0.5\% bovine serum albumin (BSA) were plated on the gel, and DMEM with $0.5 \%$ BSA and $0.5 \%$ FBS was added to the bottom of the chambers. Photographs were taken $36 \mathrm{~h}$ later to capture the cells that had invaded below the gel surface. The number of invading cells in 5 fields was counted under a $200 \times$ magnification. Each value represents the average of 3 individual experiments, and the error bars represent SD. p-values were calculated by the ANOVA test in SAS8.2 [21].

\section{Authors' contributions}

$\mathrm{XMC}$ carried out the molecular genetic studies, participated in the preparation of phagemid particles and drafted the manuscript. HLX carried out the immunoassays and performed the statistical analysis. MZL participated in transwell invasion assay. XLZ conceived of the study, and participated in its design and coordination and helped to draft the manuscript. All authors read and approved the final manuscript.

\section{Acknowledgements}

The authors thank Dr. Li ZH for his gifts of plasmids. The project is supported by the National Natural Science Foundation of China (No.

30600336) and Shanghai Leading Academic Discipline Project, Project Number: BII0.

\section{References}

I. Burg MA, Jensen-Pergakes K, Gonzalez AM, Ravey P, Baird A, Larocca $D$ : Enhanced phagemid particle gene transfer in camptothecin-treated carcinoma cells. Cancer Res 2002, 62:977-981. 
2. Larocca D, Witte A, Johnson W, Pierce GF, Baird A: Targeting bacteriophage to mammalian cell surface receptors for gene delivery. Hum Gene Ther 1998, 9:2393-2399.

3. Larocca D, Kassner PD, Witte A, Ladner RC, Pierce GF, Baird A: Gene transfer to mammalian cells using genetically targeted filamentous bacteriophage. FASEB J 1999, I3:727-734.

4. Kassner PD, Burg MA, Baird A, Larocca D: Genetic selection of phage engineered for receptor-mediated gene transfer to mammalian Cells. Biochem Biophys Res Commun 1999, 264:921-928.

5. Li ZH, Zhang J, Zhao RJ, Xu YH, Gu JR: Preparation of peptidetargeted phagemid particles using a protein III-modified helper phage. Biotechniques 2005, 39:493-497.

6. Poul MA, Marks JD: Targeted gene delivery to mammalian cells by filamentous bacteriophage. J $\mathrm{Mol}$ Biol 1999, 288:203-2II.

7. Giovine MD, Salone B, Martina Y, Amati V, Zambruno G, Cundari E, Failla CM, Saggio I: Binding properties, cell delivery and gene transfer of adenoviral penton base displaying bacteriophage. Virology 200I, 282: I02-II2.

8. Li ZH, Jiang H, Zhang J, Shi BZ, Gu JR: Cell targeted phagemid particles preparation using $E$. coli bearing ligand-pIII encoding helper phage genome. Bio Techniques 2006, 4I:706-707.

9. Liang Y, Shi B, Zhang J, Jiang H, Xu Y, Li Z, Gu J: Better gene expression by $(-)$ gene than by $(+)$ gene in phage gene delivery systems. Biotechnology Progress 2006, 22:626-630.

10. Jiang H, Cai X, Shi B, Zhang J, Li Z, Gu J: Development of efficient RNA interference system using EGF-displaying phagemid particles. Acta Pharmacologica Sinica 2008, 29:437-442.

II. Carelli S, Zadra G, Vaira V, Falleni M, Bottiglieri L, Nosotti M, Giulio AM, Gorio A, Bosari S: Up-regulation of focal adhesion kinase in non-small cell lung cancer. Lung Cancer 2006, 53:263-27I.

12. Oktay MH, Oktay K, Hamele D, Buyuk A, Koss LG: Focal adhesion kinase as a marker of malignant phenotype in breast and cervical carcinomas. Hum Pathol 2003, 34:240-245.

13. Kornberg L): Focal adhesion kinase and its potential involvement in tumor invasion and metastasis. Head Neck 1998 20:745-752.

14. Sood AK, Coffin JE, Schneider GB: Biological significance of focal adhesion kinase in ovarian cancer: role in migration and invasion. Am J Pathol 2004, 165: 1087-1095.

15. Han EK, Mcgonigal T, Wang J, Giranda VL, Luo Y: Functional analysis of focal adhesion kinase (FAK) reduction by small inhibitory RNAs. Anticancer Res 2004, 24:3899-3905.

16. Chen J, Gamou S, Takayanagi A, Ohtake Y, Ohtsubo M, Shimizu N: Receptor-mediated gene delivery using the Fab fragments of anti-epidermal growth factor receptor antibodies: improved immunogene approach. Cancer Gene Ther 1998, 5:357-364.

17. Qing K, Wang XS, Kube DM, Ponnazhagan S, Bajpai A, Srivastava A: Role of tyrosine phosphorylation of a cellular protein in adeno-associated virus 2-mediated transgene expression. Proc Natl Acad Sci USA 1997, 94:10879-10884.

18. Mitra SK, Lim ST, Chi A, Schlaepfer DD: Intrinsic focal adhesion kinase activity controls orthotopic breast carcinoma metastasis via the regulation of urokinase plasminogen activator expression in a syngeneic tumor model. Oncogene 2006, 25:4429-4440.

19. Rondot S, Koch J, Breitling F, Dübe S: A helper phage to improve single-chain antibody presentation in pahge display. Nat Biotechnol 200I, 19:75-78.

20. Zhou GF, Ye F, Cao LH, Zha XL: Over expression of integrin alpha 5 beta $I$ in human hepatocellular carcinoma cell line suppresses cell proliferation in vitro and tumorigenicity in nude mice. Mol Cell Biochem 2000, 207:49-55.

21. Cai XM, Tao BB, Wang LY, Liang YL, Jin JW, Yang Y, Hu YL, Zha XL Protein phosphatase activity of $P T E N$ inhibited the invasion of glioma cells with epidermal growth factor receptor mutation type III expression. Int J Cancer 2005, I I7:905-9|2.

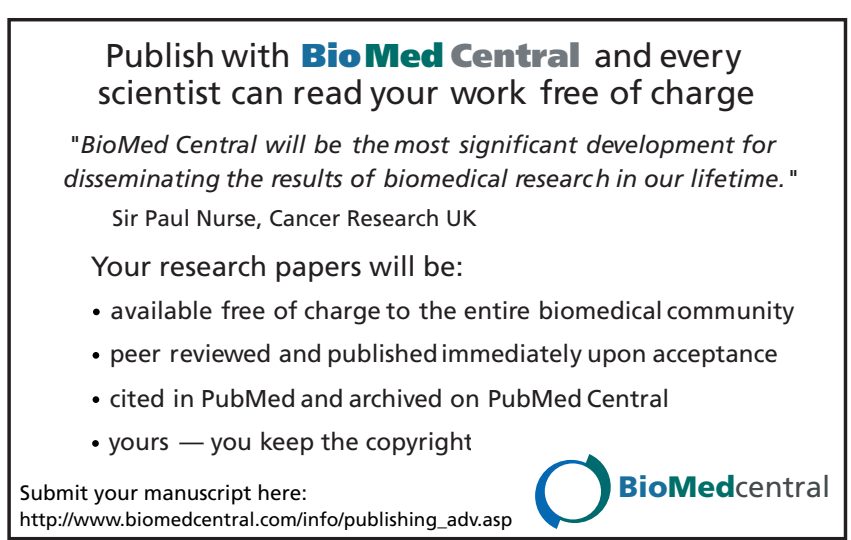

